\title{
1 Detection and molecular characterisation of SARS-CoV-2 2 in farmed mink (Neovision vision) in Poland
}

3 Lukasz Rabalski ${ }^{1} \ddagger$, Maciej Kosinski ${ }^{1}$, Teemu Smura ${ }^{2}$, Kirsi Aaltonen ${ }^{2,3}$, Ravi Kant ${ }^{2,3}$, Tarja

4 Sironen $^{2,3}$, Boguslaw Szewczyk ${ }^{1}$, Maciej Grzybek ${ }^{4}{ }^{*}$,

5

6

7

8

1 Laboratory of Recombinant Vaccines, Intercollegiate Faculty of Biotechnology of University of Gdansk and Medical University of Gdansk, Abrahama 58, 80-307, Gdansk, Poland

${ }^{2}$ Department of Virology, University of Helsinki, Haartmaninkatu 3, Helsinki FI-00290, Finland

${ }^{3}$ Department of Veterinary Biosciences, University of Helsinki, Agnes Sjöbergin katu 2, Helsinki FI-00790, Finland

${ }^{4}$ Department of Tropical Parasitology, Institute of Maritime and Tropical Medicine, Medical University of Gdansk, Powstania Styczniowego 9B, 81-519, Gdynia, Poland.

$\ddagger$ These authors contributed equally to this work.

*ADDRESS FOR CORRESPONDENCE: Maciej Grzybek, PhD, DSc; Department of Tropical Parasitology, Medical University of Gdansk, Powstania Styczniowego 9B, 81-519 Gdynia, Poland. Email: maciej.grzybek@ gumed.edu.pl Tel: + 48583491941

KEYWORDS: SARS-CoV-2; interstitial pneumonia; mink; transmission; spillover; zoonoses

\section{ABSTRACT}

SARS-CoV-2 is the aetiological agent of COVID-19 disease and has been spreading worldwide since December 2019. The virus has been shown to infect different animal species under experimental conditions. Also, minks have been found to be susceptible to SARS-CoV2 infection in fur farms in Europe and the USA. Here we investigated 91 individual minks from a farm located in Northern Poland. Using RT-PCR, antigen detection and NGS, we confirmed 15 animals positive for SARS-CoV-2. The result was verified by sequencing of full viral genomes, confirming SARS-CoV-2 infection in Polish mink. Country-scale monitoring conducted by veterinary inspection so far has not detected the presence of SARS-CoV-2 on other mink farms. Taking into consideration that Poland has a high level of positive diagnostic tests among its population, there is a high risk that more Polish mink farms become a source 
34 for SARS-CoV-2. Findings reported here and from other fur producing countries urge the 35 assessment of SARS-CoV-2 prevalence in animals bred in Polish fur farms.

\section{INTRODUCTION}

The identification of possible pathogen hosts and the study of transmission dynamics in their populations are crucial steps in controlling zoonotic diseases. In the case of SARS-CoV-2, the origin is most likely bats (Zhou et al., 2020), but the intermediate host has not been confirmed yet. SARS-CoV-2 seems to readily jump from people to other animal species, in particular carnivores, (Jo et al., 2020) raising the concern of new animal sources of COVID-19.

SARS-CoV-2 infections in mink have been reported from farms in Denmark, Netherlands, USA, Italy, Spain, Sweden, Greece and Lithuania (Hammer et al., 2021; Koopmans, 2020; Oreshkova et al., 2020; Oude Munnink et al., 2020) (Figure 1).

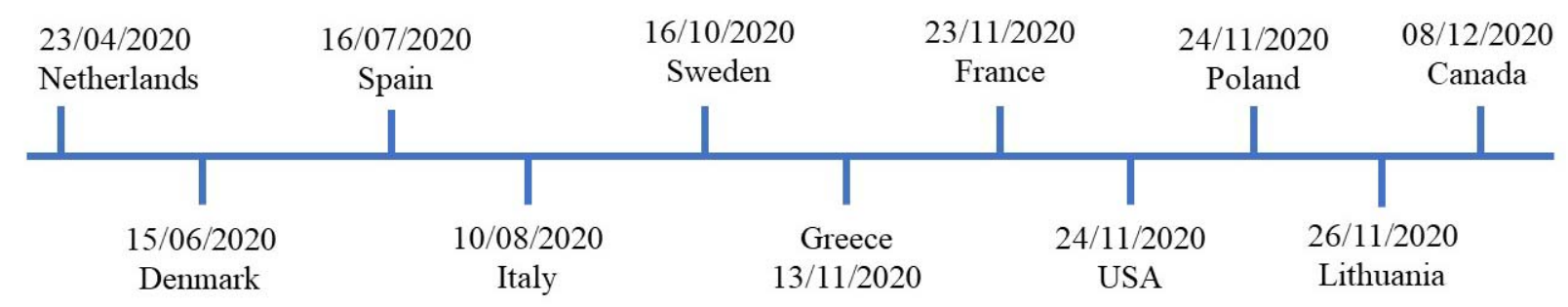

Figure 1. The time-line of SARS-CoV-2 infections in mink farms around the world, according to the World Organisation for Animal Health (World Organisation for Animal Health, 2020).

Due to SARS-CoV-2 outbreaks in mink farms and its appearance in the surrounding communities, the European Centre for Disease Prevention and Control and the WHO have emphasised the importance of surveying the host-animal interface and collaboration among virologists and epidemiologists to track and characterise viral mutations (WHO, 2020). Following SARS-CoV-2 infections in minks in the Netherlands, the Dutch Ministry of Agriculture decided to cull all minks from different farms. The Danish National Institute of Public Health announced the culling of all 17 million mink in the country after it found that the virus had spilt back from mink farms into the human community. Recent data available from Denmark and the Netherlands on these mink-associated SARS-CoV-2 variants suggest that these variants can spread rapidly in mink farms and among nearby human communities. 
59 Humans infected with the mink-related variants do not appear to have more severe clinical

60 symptoms than those infected with non-mink-related variants (Oude Munnink et al., 2020).

61 After Denmark, Poland is the second-largest producer of mink pelts in Europe. The total 62 number of fur animal farms in Poland is 810 (including fox, mink, raccoon dog, and 63 chinchillas). There are 354 active Polish mink farms with $\sim 6.3$ million individual mink. In 64 2019, Polish mink farmers sold 8.5 million mink pelts (EU Fur Association, 2020; ZPP, 65 2020).

66 As of this writing, Poland has recorded over 1135676 COVID-19 cases, with over 22864

67 total deaths $(19 / 12 / 2020$ ECDC, 2020).

Considering the recent reports of SARS-CoV-2 mink infections in other European countries and the high incidence of SARS-CoV-2 human infections in Poland, we conducted SARSCoV-2 monitoring in mink on one farm located in Pomorskie Voivodeship in North Poland.

\section{MATERIALS AND METHODS}

\subsection{Material collection}

Throat swabs (BIOCOMA, Shenzhen, China) were collected from 91 minks from a mink farm located in Pomorskie Voivodeship in Northern Poland on $17^{\text {th }}$ November 2020. The material was collected from minks culled for pelting. The farm owner reported no respiratory symptoms in the animals.

\subsection{Statistical analysis}

The $95 \%$ confidence limits were calculated using bespoke software "PERCENTAGE CONFIDENCE LIMITS VS 13" (courtesy of Dr F.S. Gilbert and Prof. J.M. Behnke, University of Nottingham), based on the statistical tables (Sokal and Rohlf, 1995)

\subsection{RNA isolation}

A total of $150 \mu \mathrm{l}$ of each sample from the swab in inactivation buffer was added to $300 \mu \mathrm{l}$ of RLT lysis buffer (RNeasy Mini kit, Qiagen, Hilden, Germany). Samples were mixed by vortexing and incubated for 10 minutes at room temperature. After incubation, $400 \mu \mathrm{l}$ of $70 \%$ ethanol was added to each sample and mixed by pipetting. The lysate was transferred to an RNeasy Mini spin column with collection tube and centrifuged 1 minute at 13000 RPM. 
91 Columns were washed once with $700 \mu \mathrm{RW} 1$ and twice with $500 \mu \mathrm{l}$ RPE. Between every

92 wash, columns were centrifuged, and flow-through was discarded. Elution was performed by

93 adding $50 \mu \mathrm{l}$ of PCR-grade water to the column and incubating for 2 minutes. Columns were

94 placed into new tubes and centrifuged at 13000 RPM for 1 minute. After isolation, samples

95 were stored for less than 2 hours at $4{ }^{\circ} \mathrm{C}$ before further steps. No human origin samples were

96 processed at the same time.

97

\subsection{Real-time RT-PCR}

99 For each sample, the reaction mixture was prepared using a polymerase, water and primers

100 and probes (Corman et al., 2020) in white 8-well q-PCR strips with optical clear caps

101 according to Table 1.

102

103

\begin{tabular}{|c|c|}
\hline qPCR grade water & $8 \mu \mathrm{l}$ \\
\hline 4x TaqPath 1 step RT-PCR polymerase & $5 \mu \mathrm{l}$ \\
\hline Forward primer Rdrp gene & \multirow{3}{*}{$1 \mu \mathrm{l}$} \\
\hline Reverse primer RdRp gene & \\
\hline Rdrp gene probe (FAM) & \\
\hline Forward primer E gene & \multirow{3}{*}{$1 \mu \mathrm{l}$} \\
\hline Reverse primer E gene & \\
\hline E gene probe (HEX) & \\
\hline Sample & $5 \mu l$ \\
\hline Volume of reaction & $20 \mu 1$ \\
\hline
\end{tabular}


105 Positive control and no template control (NTC) reactions were also prepared. Reactions were 106 mixed and loaded into Light Cycler 480 (Applied Biosystems, Foster City, California, United 107 States) with the program described below (Table 2). After each cycle of amplification, the 108 signal from each sample was measured in both FAM (Rdrp gene) and HEX (E gene) 109 channels.

\begin{tabular}{|l|l|l|l|}
\hline Step & No. of cycles & Temperature & Time \\
\hline UNG incubation & 1 & $25^{\circ} \mathrm{C}$ & 2 minutes \\
\hline RT incubation & 1 & $50{ }^{\circ} \mathrm{C}$ & 15 minutes \\
\hline Enzyme activation & 1 & $95^{\circ} \mathrm{C}$ & 2 minutes \\
\hline Amplification & 40 & $95^{\circ} \mathrm{C}$ & 3 seconds \\
\cline { 3 - 5 } & & $60{ }^{\circ} \mathrm{C}$ & 30 seconds \\
\hline
\end{tabular}

Table 2. The program used for RT-qPCR.

\subsection{SARS-CoV-2 antigen detection in mink}

114 We performed two different antigen tests to confirm the presence of virus antigen either in the swab or the serum samples.

\subsubsection{Antigen test 1 for the swab samples}

117

118 Antigen tests were conducted on samples positive in qPCR. Three negative samples were 119 used as control. A total of $150 \mu \mathrm{l}$ of transport medium from each swab was transferred to 120 tubes from a COVID-19 Antigen Detection Kit (Zhuhai Lituo Biotechnology CO., LTD.) 121 containing an extraction buffer. Samples were mixed and incubated for 1 minute. Two drops 
122 of each sample were added to the sample window on test cassettes. Results were read after 12

123 minutes.

124

125

126

127

128

129

130

131

132

133

134

135

136

137

138

139

140

141

142

143

144

145

146

147

148

149

150

151

152

153

\subsubsection{Antigen test 2 for the serum samples}

Ninety-one mink serum samples were tested with SARS-CoV-2 antigen ELISA (COV-04-S, Salofa Oy, Finland) according to the kit instructions. This test is a double-antibody sandwich ELISA. The results were obtained according to the formula based on the concentration standards provided in the kit. The cut-off value for this test was given as $2.97 \mathrm{pg} / \mathrm{ml}$. The tests were repeated twice, and additional dilutions were performed to determine the final concentration as suggested in the kit instructions.

\subsection{Full SARS-CoV-2 genome sequencing and classification}

SARS-CoV-2 genome sequencing was performed at the Medical University of Gdansk, University of Gdansk, Poland and the University of Helsinki, Finland using samples containing RNA isolated from positive swabs (amplification of two target genes in RT-PCR) or inconclusive (only single target gene amplification). At Gdansk two independent protocols were used for SARS-CoV-2 genome sequencing: Illumina RNA prep with enrichment for respiratory virus oligos panel V2 followed by Illumina MiniSeq medium output run that produced 150-nucleotide paired-end reads, and ARTICv3 amplicon generation followed by Oxford Nanopore Technology MinION run (Quick 2020). No human origin samples were processed at the same time. No DNA / rRNA depletion methods were used. Reads were basecalled, debarcoded and trimmed to delete adapter, barcode and PCR primer sequences. The fasta files generated by the Illumina procedure were further analysed in Kraken2 software to classify every read to reference database containing viral and American mink genomes (Wood et al., 2019).

In Helsinki, the sequencing libraries were prepared using Illumina DNA prep kit (New England BioLabs). The library fragment sizes were measured using agarose gel electrophoresis and the concentrations using Qubit dsDNA HS Assay Kit (Life Technologies, Carlsbad, California, USA) and NEBNext Library Quant Kit for Illumina (New England BioLabs, Ipswich, Massachusetts, USA). Sequencing was conducted using MiSeq V3 reagent kit with 250 bp reads. Raw sequence reads were trimmed and low quality (quality score <30) 
154

155

156

157

158

159

160

161

162

163

164

165

166

167

168

169

170

171

172

173

174

175

176

177

178

179

180

181

182

183

and short (<50 nt) sequences removed using Trimmomatic (Bolger et al., 2014). The trimmed sequence reads were assembled against SARS-CoV-2 reference sequence (NC_045512.2) using BWA-MEM algorithm ( $\mathrm{Li}$ 2013) implemented in SAMTools version 1.8 (Li et al., 2009).

\subsection{Phylogenetic analysis of SARS-CoV-2 isolates}

The dataset consisted of all genetic sequences of SARS-CoV-2 from this research, Poland, Germany, Lithuania, Latvia, Estonia, Russia, and Ukraine completed with representative pool "Europe" by Nexstrain.org (https://nextstrain.org/ncov/europe) resulting in a total number of 5778 entries. Phylogenetic analysis was performed using the procedure recommended by Nextstrain.org with modifications in subsampling region filtering procedure, where the number of sequences per country was 40 (Hadfield et al., 2018). In short: the Augur toolkit v10.1.1 was used for phylogenetic analysis and Auspice v2.10.1 for visualisation. Possible time of divergence for samples was inferred using the TreeTime pipeline implemented in Nextsrain analysis and presented on the phylogenetic tree (Sagulenko et al., 2018).

\subsection{Ethical Approval}

This study was carried out with due regard for the principles required by the European Union and the Polish Law on Animal Protection. No permit from Local Bioethical Committee for Animal Experimentation was obtained because animals were culled by the owner for production of pelts. Samples were collected post mortem.

\section{RESULTS}

\subsection{Prevalence of SARS-CoV-2}

Using two targets RT-PCR, antigen detection and NGS, we confirmed 15 SARS-CoV-2 positive individuals (16.5\% [8.4-28.6]). Out of the 91 samples, 17 showed a positive RT-PCR result in the SARS-CoV-2 E gene amplification (18.7\% [10.3-31.1]). Eight of these were also positive for the RdRp gene (8.8\% [3.5-19.3]).

Table 3 summarises the results for the applied diagnostic approach. Highlighted samples are considered SARS-CoV-2 positive.

Table 3. Results of different techniques that confirm the detection of SARS-CoV-2 in samples collected from farmed minks in Poland. Column 1 - individual sample name; column 
1842 and 3 - RT-qPCR assay; column 4 - antigen detection assay in swab; column 5 - antigen

185 detection assay in serum; column 6 - full viral genome sequencing. Highlighted samples are

186 considered positive for SARS-Cov-2 by nucleic acid amplification technique, serology or

187 NGS. Cp-crossing point, RdRp - RNA dependent RNA polymerase, E - Envelope small

188 membrane protein, $\mathrm{N}$ - Nucleoprotein, $\mathrm{sp}$ - strong positive, $\mathrm{p}$ - positive, $\mathrm{n}$ - negative, nc - not checked,

NTC

no

template

control. 


\begin{tabular}{|c|c|c|c|c|c|}
\hline Sample Name & Cp for gene $\mathrm{RdRp}$ & $\mathrm{Cp}$ for gene $\mathrm{E}$ & $\begin{array}{l}\text { The antigen in swab } \\
\text { (strong pos/pos/neg) }\end{array}$ & $\begin{array}{l}\text { The antigen in serum } \\
\text { (concentration of } \mathrm{N} \text { protein) }\end{array}$ & $\begin{array}{l}\text { Sequence obtained } \\
\text { (full/partial/nc) }\end{array}$ \\
\hline mink_4 & - & 24.90 & $\mathrm{sp}$ & 48.68 & full \\
\hline mink_5 & 24.36 & 16.88 & $\mathrm{sp}$ & 3.3 & full \\
\hline mink_6 & - & 34.88 & $\mathrm{n}$ & & partial \\
\hline mink_20 & - & - & $\mathrm{p}$ & 132.24 & partial \\
\hline mink_27 & - & 35.05 & $\mathrm{n}$ & & partial \\
\hline mink_36 & - & - & $\mathrm{p}$ & 271.3 & partial \\
\hline mink_39 & - & - & $\mathrm{n}$ & 35.59 & partial \\
\hline mink_42 & - & 25.62 & $\mathrm{p}$ & & full \\
\hline mink_46 & 34.93 & 22.75 & $\mathrm{p}$ & & full \\
\hline mink_48 & 29.89 & 22.52 & $\mathrm{sp}$ & & full \\
\hline mink_49 & - & 27.79 & $\mathrm{p}$ & & full \\
\hline
\end{tabular}




\begin{tabular}{|c|c|c|c|c|c|}
\hline mink_50 & 32.39 & 21.61 & $\mathrm{sp}$ & & full \\
\hline mink_63 & - & - & $\mathrm{n}$ & 19.3 & partial \\
\hline mink_67 & 36.88 & 23.34 & $\mathrm{p}$ & & full \\
\hline mink_70 & - & 24.40 & $\mathrm{p}$ & & partial \\
\hline mink_76 & 32.53 & 22.73 & $\mathrm{p}$ & & full \\
\hline mink_77 & 32.87 & 20.3 & $\mathrm{sp}$ & & full \\
\hline mink_83 & 15.89 & 21.27 & $\mathrm{sp}$ & 91.57 & full \\
\hline mink_88 & - & 33.99 & $\mathrm{p}$ & & full \\
\hline mink_89 & - & 35.39 & $\mathrm{n}$ & & $\mathrm{nc}$ \\
\hline mink_90 & - & 35.61 & $\mathrm{n}$ & & $\mathrm{nc}$ \\
\hline NTC & - & - & & & \\
\hline Positive control & 16.91 & 23.31 & & & \\
\hline
\end{tabular}


191

192

193

194

195

196

197

198

199

200

201

202

203

204

205

206

207

208

209

210

211

212

213

214

215

\subsection{SARS-CoV-2 antigens detected in mink}

\subsubsection{Antigen test from the swab}

Samples mink_4, mink_5, mink_48, mink_50, mink_77 and mink_83 gave highly visible signals in both control and test lines. Samples mink_20, mink_36, mink_42, mink_46, mink_49, mink_67, mink_76 and mink_88 gave next to a highly visible control line a much less pronounced test line. In all other samples, only the test line was visible. All eight realtime RT-PCR positive samples were also positive in the antigen test. Additionally, five E gene-positive samples were also positive in the antigen test, but four were negative. In the sample mink_20 that was positive in the antigen test, SARS-CoV-2 RNA was not detected by RT-PCR.

\subsubsection{Reads classification and SARS-CoV-2 genome sequences}

The final validation of the detection of SARS-CoV-2 in minks was a classification of nextgeneration sequencing reads to the database containing reference viral, human and American mink genomes. Three independent approaches were used to achieve full virus genomes, and the data resulting from procedures are presented in Table 4. Only samples that gave rise to a complete SARS-CoV-2 genome sequence are shown. The number of Illumina reads generated for samples mink_4, mink_42, mink_49, mink_76 and mink_88 were not enough to produce full SARS-CoV-2 genomes. For these samples, genomes were obtained by ARTIC procedure.

Table 4. Total mean coverage (ONT and Illumina reads) for every sample that full SARSCoV-2 genome was established. Last three columns represent how many Illumina reads were generated for each sample and how many of them were classified to SARS-CoV-2 or American mink genomes.

\begin{tabular}{|c|c|c|c|c|}
\hline Sample name & Mean coverage & $\begin{array}{c}\text { Total Illumina } \\
\text { reads }\end{array}$ & $\begin{array}{c}\text { SARS-CoV-2 } \\
\text { reads }\end{array}$ & Mink reads \\
\hline mink_4 & 949.6 & 152609 & 37 & 131204 \\
\hline mink_5 & 2776 & 396259 & 159484 & 184135 \\
\hline mink_42 & 847.8 & 66515 & 868 & 34968 \\
\hline
\end{tabular}




\begin{tabular}{|c|c|c|c|c|}
\hline mink_46 & 1039.5 & 387682 & 25975 & 241788 \\
\hline mink_48 & 1151 & 125328 & 9526 & 64363 \\
\hline mink_49 & 394.6 & 487771 & 1711 & 348017 \\
\hline mink_50 & 1839.6 & 425333 & 79589 & 246546 \\
\hline mink_67 & 882.1 & 167963 & 10834 & 114163 \\
\hline mink_76 & 723 & 99660 & 2105 & 77945 \\
\hline mink_77 & 16078.5 & 2424311 & 1690172 & 662381 \\
\hline mink_83 & 111978.9 & 13495934 & 12730431 & 611926 \\
\hline mink_88 & 1205.2 & 1071003 & 2822 & 611787 \\
\hline
\end{tabular}

The 12 mink originated SARS-CoV-2 sequences were checked for the presence of mink specific mutations detected earlier in minks from the Netherlands and Denmark, but none were found, pointing towards a recent introduction of SARS-CoV2 in Polish minks (Figure $1)$.

222 The alignment of the full genome sequence from 12 individual samples showed multiple 223 polymorphisms on different nucleotide sites. Many of them gave rise to changes in amino 224 acid in comparison to the reference (MN908947). Two specific mutations present in all 225 samples were found in the S protein: G75V and C1247F. These mutations are present in 199 226 for S: G75V and 83 for S: C1247F isolates previously published in GISAID. Other rear 227 amino acid variants present in every Polish mink SARS-CoV-2 isolate were found in five more proteins: nsp2, nsp3, nsp14, nsp15 and $\mathrm{N}$.

229 Based on the dataset (Material and methods 2.6.) we inferred phylogenetic relationship by 230 estimating divergence times between every isolate (Figure 1). The analysis estimates that the 231 most recent common ancestor for Polish minks SARS-CoV-2 and two most similar sequences 232 (German/NW-HHU-340/2020 and Norway/4235/2020) diverged around $31^{\text {st }}$ September 2020. 233 Multiple mutations in amino acid sequences were recognised. If the molecular evolution starts 234 after the virus introduction to the farm, this incident is estimated to happen around 4th 235 October 2020. 


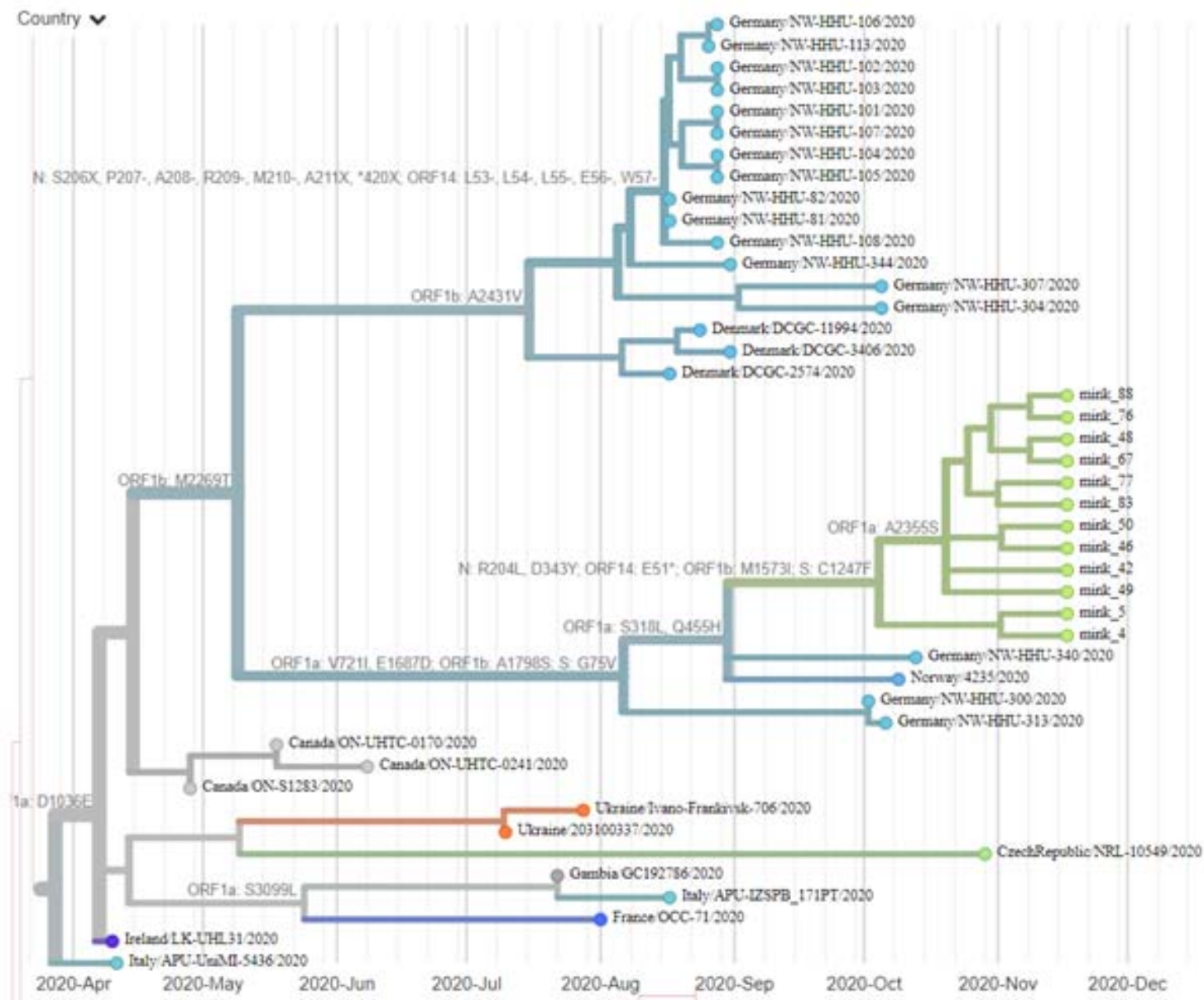

Figure 1. Phylogenetic tree estimating divergence time presenting only closely related isolates that were included in the dataset. Indications on branches represent amino acid changes that were associated with divergence. Produced using Nextstrain.org.

\section{DISCUSSION}

243 Identifying new animal species that can serve as animal sources of SARS-CoV-2 and 244 predicting where novel outbreaks are most likely to occur are crucial steps for preventing and 245 minimising the extent of SARS-CoV-2 infections among humans (Hemida and Ba Abduallah, 246 2020). Recent reports confirmed the presence of SARS-CoV-2 in different animal species,

247 including fur animals (i.e. minks and racoon dogs) (Abdel-Moneim and Abdelwhab, 2020;

248 Freuling et al., 2020). 
Here we report the presence of SARS-CoV-2 in minks from a fur farm in Northern Poland. We report a $16.5 \%$ prevalence of SARS-CoV-2 in the tested minks and confirm the presence of SARS-CoV-2 in farmed minks in Poland.

Poland is one of the largest fur producers in Europe. Considering the number of farmed minks in the country and the significant number of people employed in this sector, we seek to raise awareness in the scientific community and the mink industry that minks are susceptible to SARS-CoV-2 infection.

Previous studies reported the detection of viral RNA in airborne inhalable dust in mink farms (Oreshkova et al., 2020). Moreover, close contact of farmworkers with animals during feeding, culling, and dehiding increase the risk of exposure.

We believe that a country-scale biomonitoring programme should be activated as soon as possible to prevent the fur production sector from being a reservoir for future spillover of SARS-CoV-2 to humans. Samples for molecular diagnostics should be obtained for all farms in Poland following the highest standards of material collection, sample handling, and molecular detection of SARS-CoV-2.

We report a possible rise of the new genotype that possesses sporadic mutations through the full genome sequence. Two mutations located in Spike protein (G75V and C1247F) are present in all Polish mink virus isolates. G75V mutation is localised in the NTD domain and could be responsible for interaction with host receptors or stabilising Spike protein in a constrained prefusion state (Arya et al., 2021). Non-other sequences of SARS-CoV-2 deposited to date in GISAID possess these two mutations simultaneously (Shu and McCauley, 2017). For now, we do not know if this possible genotype has the ability for transmission to humans. Wide monitoring of humans living in close surroundings to the mink farm should answer this question.

\section{REFERENCES:}

Arya, R., Kumari, S., Pandey, B., Mistry, H., Bihani, S.C., Das, A., Prashar, V., Gupta, G.D., Panicker, L., Kumar, M., 2021. Structural insights into SARS-CoV-2 proteins. J. Mol. Biol. 433, 166725. https://doi.org/10.1016/j.jmb.2020.11.024

Bolger, A.M., Lohse, M., Usadel, B., 2014. Trimmomatic: a flexible trimmer for Illumina sequence data. Bioinformatics 30, 2114-2120. https://doi.org/10.1093/bioinformatics/btu170 
ECDC, 2020. COVID-19 situation update for the EU/EEA and the UK [WWW Document]. URL https://www.ecdc.europa.eu/en/cases-2019-ncov-eueea (accessed 12.19.20).

EU Fur Association, 2020. EU Fur Association [WWW Document]. URL https://www.sustainablefur.com/ (accessed 12.13.20).

Hadfield, J., Megill, C., Bell, S.M., Huddleston, J., Potter, B., Callender, C., Sagulenko, P., Bedford, T., Neher, R.A., 2018. Nextstrain: real-time tracking of pathogen evolution. Bioinformatics 34, 4121-4123. https://doi.org/10.1093/bioinformatics/bty407

Hammer, A.S., Quaade, M.L., Rasmussen, T.B., Fonager, J., Rasmussen, M., Mundbjerg, K., Lohse, L., Strandbygaard, B., Jørgensen, C.S., Alfaro-Núñez, A., Rosenstierne, M.W., Boklund, A., Halasa, T., Fomsgaard, A., Belsham, G.J., Bøtner, A., 2021. SARS-CoV-2 Transmission between Mink ( Neovison vison ) and Humans, Denmark. Emerg. Infect. Dis. 27. https://doi.org/10.3201/eid2702.203794

Hemida, M.G., Ba Abduallah, M.M., 2020. The SARS-CoV-2 outbreak from a one health perspective. One Heal. 10, 100127. https://doi.org/10.1016/j.onehlt.2020.100127

Jo, W.K., Oliveira $\square$ Filho, E.F., Rasche, A., Greenwood, A.D., Osterrieder, K., Drexler, J.F., 2020. Potential zoonotic sources of SARS $\square \mathrm{CoV} \square 2$ infections. Transbound. Emerg. Dis. tbed.13872. https://doi.org/10.1111/tbed.13872

Koopmans, M., 2020. SARS-CoV-2 and the human-animal interface: outbreaks on mink farms. Lancet Infect. Dis. 1. https://doi.org/10.1016/S1473-3099(20)30912-9

Li, H., Handsaker, B., Wysoker, A., Fennell, T., Ruan, J., Homer, N., Marth, G., Abecasis, G., Durbin, R., 2009. The Sequence Alignment/Map format and SAMtools. Bioinformatics 25, 2078-2079. https://doi.org/10.1093/bioinformatics/btp352

Oreshkova, N., Molenaar, R.J., Vreman, S., Harders, F., Oude Munnink, B.B., Hakze-van der Honing, R.W., Gerhards, N., Tolsma, P., Bouwstra, R., Sikkema, R.S., Tacken, M.G., de Rooij, M.M., Weesendorp, E., Engelsma, M.Y., Bruschke, C.J., Smit, L.A., Koopmans, M., van der Poel, W.H., Stegeman, A., 2020. SARS-CoV-2 infection in farmed minks, the Netherlands, April and May 2020. Eurosurveillance 25. https://doi.org/10.2807/15607917.ES.2020.25.23.2001005

Oude Munnink, B.B., Sikkema, R.S., Nieuwenhuijse, D.F., Molenaar, R.J., Munger, E., Molenkamp, R., van der Spek, A., Tolsma, P., Rietveld, A., Brouwer, M., BouwmeesterVincken, N., Harders, F., Hakze-van der Honing, R., Wegdam-Blans, M.C.A., Bouwstra, R.J., GeurtsvanKessel, C., van der Eijk, A.A., Velkers, F.C., Smit, L.A.M., Stegeman, A., van der Poel, W.H.M., Koopmans, M.P.G., 2020. Transmission of SARS-CoV-2 on mink farms between humans and mink and back to humans. Science (80-. ). eabe5901. https://doi.org/10.1126/science.abe5901

Sagulenko, P., Puller, V., Neher, R.A., 2018. TreeTime: Maximum-likelihood phylodynamic analysis. Virus Evol. 4. https://doi.org/10.1093/ve/vex042

Shu, Y., McCauley, J., 2017. GISAID: Global initiative on sharing all influenza data - from vision to reality. Eurosurveillance 22. https://doi.org/10.2807/15607917.ES.2017.22.13.30494 
Sokal, R.R., Rohlf, F.J., 1995. Statistical Tables. W. H. Freeman, New York.

WHO, 2020. WHO-convened Global Study of the Origins of SARS-CoV-2 [WWW Document]. WHO. URL https://www.who.int/publications/m/item/who-convenedglobal-study-of-the-origins-of-sars-cov-2 (accessed 12.13.20).

Wood, D.E., Lu, J., Langmead, B., 2019. Improved metagenomic analysis with Kraken 2. Genome Biol. 20, 257. https://doi.org/10.1186/s13059-019-1891-0

World Organisation for Animal Health, 2020. World Organisation for Animal Health [WWW Document]. URL https://www.oie.int/en/scientific-expertise/specific-information-andrecommendations/questions-and-answers-on-2019novel-coronavirus/events-in-animals/ (accessed 12.19.20).

Zhou, P., Yang, X.-L., Wang, X.-G., Hu, B., Zhang, L., Zhang, W., Si, H.-R., Zhu, Y., Li, B., Huang, C.-L., Chen, H.-D., Chen, J., Luo, Y., Guo, H., Jiang, R.-D., Liu, M.-Q., Chen, Y., Shen, X.-R., Wang, X., Zheng, X.-S., Zhao, K., Chen, Q.-J., Deng, F., Liu, L.-L., Yan, B., Zhan, F.-X., Wang, Y.-Y., Xiao, G.-F., Shi, Z.-L., 2020. A pneumonia outbreak associated with a new coronavirus of probable bat origin. Nature 579, 270-273. https://doi.org/10.1038/s41586-020-2012-7

ZPP, 2020. Fakty o hodowli zwierzat futerkowych w Polsce. Warsaw.

\section{Data availability}

The complete genome sequences of SARS-CoV-2 isolated from farmed Polish minks have been deposited in GISAID under the accession numbers EPI_ISL_732948 - EPI_ISL_732959.

\section{Acknowledgements}

We gratefully acknowledge the following Authors from the Originating laboratories responsible for obtaining the specimens and the Submitting laboratories where genetic sequence data were generated and shared via the GISAID Initiative, on which this research is partially based (Supplementary Table 1). MG thanks Alicja Rost, Ewa Zieliniewicz and Karolina Baranowicz for their assistance in the laboratory. LR thanks Bartosz Wasag for helping in sequencing. We thank veterinary surgeons for their help in samples collection.

\section{Conflicts of Interest}

The authors declare no conflict of interests. 


\section{Funding:}

355 We appreciate the support from the University of Gdańsk, Medical University of Gdańsk and

356 University of Helsinki. M.G. was supported by the National Science Centre, Poland under the

357 BiodivERsA3 program - BioRodDis_COVID-19 (2019/31/Z/NZ8/04028). L.R. was

358 supported by the Ministry of Science and Higher Education. Decision No. 54 / WFSN / 2020.

359 "Co-infections with SARS-CoV-2, Database of COVID-19 Accompanying Infections". This

360 study was supported by the VEO - European Union's Horizon 2020 (grant number 874735)

361 and the Jane and Aatos Erkko Foundation. 